\title{
Modeling the Glomerular Filtration Barrier and Intercellular Crosstalk
}

\author{
Kerstin Ebefors ${ }^{1 \dagger}$, Emelie Lassén ${ }^{2 \dagger}$, Nanditha Anandakrishnan ${ }^{2}$, Evren U. Azeloglu ${ }^{2}$ and \\ Ilse S. Daehn ${ }^{2 *}$ \\ ${ }^{1}$ Department of Physiology, Institute of Neuroscience and Physiology, Sahlgrenska Academy, University of Gothenburg, \\ Gothenburg, Sweden, 'Division of Nephrology, Department of Medicine, Icahn School of Medicine at Mount Sinai, \\ New York, NY, United States
}

The glomerulus is a compact cluster of capillaries responsible for blood filtration and initiating urine production in the renal nephrons. A trilaminar structure in the capillary wall forms the glomerular filtration barrier (GFB), composed of glycocalyx-enriched and fenestrated endothelial cells adhering to the glomerular basement membrane and specialized visceral epithelial cells, podocytes, forming the outermost layer with a molecular slit diaphragm between their interdigitating foot processes. The unique dynamic and selective nature of blood filtration to produce urine requires the functionality of each of the GFB components, and hence, mimicking the glomerular filter in vitro has been challenging, though critical for various research applications and drug screening. Research efforts in the past few years have transformed our understanding of the structure and multifaceted roles of the cells and their intricate crosstalk in development and disease pathogenesis. In this review, we present a new wave of technologies that include glomerulus-on-a-chip, three-dimensional microfluidic models, and organoids all promising to improve our understanding of glomerular biology and to enable the development of GFB-targeted therapies. Here, we also outline the challenges and the opportunities of these emerging biomimetic systems that aim to recapitulate the complex glomerular filter, and the evolving perspectives on the sophisticated repertoire of cellular signaling that comprise the glomerular milieu.

\footnotetext{
Keywords: glomerular filtration barrier, crosstalk, in vitro, podocyte, glomerular endothelial cell, 3D model

"A model is a lie that helps you see the truth" - Dr. Howard Skipper
}

\section{INTRODUCTION}

The glomerular filtration barrier (GFB) is a highly specialized interface responsible for blood filtration that is charge and size selective. While its functionality and integrity are maintained by a constant interaction between glomerular endothelial cells (GECs), the glomerular basement membrane (GBM), and podocytes (Rennke et al., 1975; Rennke and Venkatachalam, 1979), they are also influenced by the milieu and dynamics of the renal blood flow. In glomerular diseases, this barrier loses functional integrity, allowing the passage 
of macromolecules and cells, and results in morphological changes, increasing the risk of long-term kidney damage that ultimately leads to kidney failure (USRDS, 2020). This is a growing worldwide health problem that accounts for a substantial economic burden (Honeycutt et al., 2013). Although the etiologies differ among glomerular diseases, damage to the GFB often has the same clinical manifestations, proteinuria or hematuria, and impaired glomerular filtration rate (GFR).

The interconnectivity and structural complexity of the GFB have favored the use of experimental in vivo models, where these traits are preserved. Using rodent models is regarded as the gold standard in GFB research. Mice have been used extensively to study the GFB, given the advantage that the complexity of the GFB microenvironment can be fully recapitulated, that there are several available genetically defined strains and the relative ease of single gene targeting (Becker and Hewitson, 2013). Also, transgenic lines with fluorescent reporters in different glomerular cell types provide visual readout and have been useful for determining the origins and fate of glomerular cells in vivo (Hackl et al., 2013). There are however significant challenges with mimicking human disease in animals, as many models do not completely recapitulate human disease manifestations and instead allow only for studies of certain disease aspects (Becker and Hewitson, 2013). However, the use of animal models is of particular importance for pharmacodynamics and pharmacokinetics testing, where the effects of pharmaceutical interventions can be examined at the systemic level to determine drug safety and efficacy before entering human trials.

The use of transgenic zebrafish strains is growing as a vertebrate model for GFB research (Zhou and Hildebrandt, 2012; Hansen et al., 2020) and has proven to be a useful tool to investigate glomerular disease development and the effects of drugs on GFB (Schiffer et al., 2015; Müller-Deile et al., 2019). Although studies in zebrafish are more timeand cost-efficient compared with rodent models, there are some inherent caveats. It can for instance be difficult to detect proteinuria or the clearance of specific markers of interest in the urine due to the surrounding water volume. Also, zebrafish have numerous duplicate genes (Woods et al., 2000), which complicates the generation of knockout strains, and they also have the ability to regenerate nephrons de novo after injury. Other limitations include the need for microinjections to the dorsal aorta and cardinal vein for certain drugs, which limits throughput. Altogether, animal work can be expensive, has limited throughput, and poses challenges for studying intricate crosstalk between the cells in the glomerulus. Therefore, there is a need for microphysiological systems that can recapitulate the form and function of the GFB and offer a controlled environment for studies of isolated pathological events. Current model systems range from simple to physiologically complex and offer opportunities for examining specific mechanisms involved in the maintenance as well as damage to the GFB (Table 1). Here, we review and discuss some of the current and future experimental in vitro model systems for studying the GFB.

\section{THE FUNCTIONAL BARRIER}

The glomerulus is the filtering part of the nephron (Figure 1A) and consists of three different cell types: podocytes (visceral epithelial cells), GECs, and mesangial cells. The filtrate from the glomerulus enters the Bowman's capsule as pre-urine before reabsorption and secretion in the tubular system. Glomerular cells are highly specialized and interdependent, with fenestrated GECs covering the luminal surface of glomerular capillaries, in direct contact with the blood. Podocytes tightly wrap around the glomerular capillary vessels, with interdigitating foot processes bridged by a slit diaphragm (Figure 1B). GECs and podocytes share a common extracellular matrix (ECM), the glomerular basement membrane (GBM), and together, they form the GFB (Figure 1C). Between the capillaries are contractile mesangial cells surrounded by their ECM, providing structural support to the glomerular tuft (Brenner et al., 1978).

The GFB function relies on its three layers: podocytes, GBM, and GECs (Figure 1C). Podocytes are terminally differentiated epithelial cells that form the architectural backbone of the GFB anchored to the GBM through transmembrane receptors, such as integrins (e.g., integrin $\alpha 3$ and laminin $\beta 2$ ) and dystroglycan, and cover the outer aspect of the glomerular capillary (Pozzi et al., 2008; Meyrier, 2011). They have specialized projections that interdigitate to form the slit diaphragm, a key element in the GFB (Perico et al., 2016). The slit diaphragm proteins (e.g., nephrin and podocin) anchor to the cytoskeleton at the plasma membrane and form bridging structures between the interdigitating podocyte projections (foot processes; Kestila et al., 1998; Boute et al., 2000). Additional proteins that maintain slit diaphragm proteins, such as CD2AP, play vital roles in GFB maintenance. Podocytes are essential in GFB function, underscored by the discovery of pathogenic mutations to proteins involved in maintaining podocyte structure that are causal to proteinuric forms of kidney disease (Vivante and Hildebrandt, 2016; Li et al., 2020a).

The GBM is formed by secreted products from both podocytes and endothelial cells during glomerulogenesis (St John and Abrahamson, 2001). Its role in the barrier function is highlighted by genetic studies showing that mutations in key components of GBM; encoding laminin- $\alpha 5$ and COL4A5, or recessive COL4A3/4, results in basement membrane nephropathy due to the absence or inadequate assembly of all collagen chains. These mutations contribute to the development of nephrotic syndrome in pediatric patients and Alport syndrome, respectively (Tryggvason et al., 1993; Kashtan, 1999; Quinlan and Rheault, 2021).

Glomerular endothelial cells are highly specialized cells with fenestrae and a charged luminal endothelial surface layer, or glycocalyx, that is composed of negatively charged networks of proteoglycans, glycoproteins, and glycolipids (Ballermann, 2007; Fogo and Kon, 2010; Haraldsson and Nystrom, 2012; Khramova et al., 2021) that together with the GBM contribute to the maintenance of a charge-selective barrier which is important to restrain albumin from the glomerular filtrate (Jeansson et al., 2009; Singh et al., 2011; Öberg and Rippe, 2013; Boels et al., 2016; Figure 1C). GEC dysfunction can initiate and contribute to GFB breakdown (Haraldsson et al., 2008; Haraldsson and Nystrom, 2012; Sun et al., 2013; Daehn, 2018). In addition, activated podocytes have been shown to influence endothelial 
TABLE 1 | Comparison of in vivo and in vitro models currently used or under development for studies of the glomerular filtration barrier (GFB).

\begin{tabular}{|c|c|c|c|c|c|}
\hline & \multicolumn{4}{|c|}{ In vitro } & \multirow{2}{*}{$\begin{array}{l}\text { In vivo } \\
\text { Animal models }\end{array}$} \\
\hline & 2D monolayer & $\begin{array}{l}\text { Static } \\
\text { co-culture }\end{array}$ & $\begin{array}{l}\text { Microfluidic } \\
\text { co-culture }\end{array}$ & $\begin{array}{l}\text { Spheroids } \\
\text { organoids }\end{array}$ & \\
\hline All GFB cell types (Nishinakamura, 2019) & No & No & No & No & Yes \\
\hline $\begin{array}{l}\text { GBM (Slater et al., 2011; Chew and Lennon, 2018; } \\
\text { Hale et al., 2018; Petrosyan et al., 2019) }\end{array}$ & No & Limited & Limited & Limited & Yes \\
\hline $\begin{array}{l}\text { Glycocalyx (Singh et al., 2007; Petrosyan et al., } \\
\text { 2019; Koning et al., 2020) }\end{array}$ & Limited & Limited & Yes & Limited & Yes \\
\hline $\begin{array}{l}\text { Allows cell differentiation (relevant phenotype; Musah } \\
\text { et al., 2017; Bao et al., 2018; Nishinakamura, 2019; } \\
\text { Veissi et al., 2020) }\end{array}$ & No & No & Limited & Limited & Yes \\
\hline $\begin{array}{l}\text { Permselectivity (Li et al., 2016; Petrosyan et al., } \\
\text { 2019; Li et al., 2020b) }\end{array}$ & No & Yes & Yes & Limited & Yes \\
\hline $\begin{array}{l}\text { Recapitulation of microenvironment (Huh et al., 2013; } \\
\text { Bhatia and Ingber, 2014; Veissi et al., 2020) }\end{array}$ & No & Limited & Limited & Limited & Yes \\
\hline $\begin{array}{l}\text { Controlled microenvironment (Anandakrishnan and } \\
\text { Azeloglu, 2020) }\end{array}$ & Yes & Yes & Yes & Yes & No \\
\hline $\begin{array}{l}\text { Shear stress (Slater et al., 2012; Musah et al., 2017; } \\
\text { Yang et al., 2017; Homan et al., 2019) }\end{array}$ & No & Limited & Yes & Limited & Yes \\
\hline $\begin{array}{l}\text { Bidirectional crosstalk (Li et al., 2016; Casalena et al., } \\
\text { 2020; Veissi et al., 2020) }\end{array}$ & No & Yes & Yes & Limited & Yes \\
\hline $\begin{array}{l}\text { Material of human origin (Little and Takasato, 2015; } \\
\text { Musah et al., 2017; Anandakrishnan and Azeloglu, } \\
\text { 2020) }\end{array}$ & Yes & Yes & Yes & Yes & No \\
\hline $\begin{array}{l}\text { High throughput (Boreström et al., 2018; } \\
\text { Anandakrishnan and Azeloglu, 2020) }\end{array}$ & Yes & Limited & Limited & Limited & No \\
\hline $\begin{array}{l}\text { Development of personalized/precision medicine } \\
\text { (Anandakrishnan and Azeloglu, 2020) }\end{array}$ & Yes & Yes & Yes & Yes & No \\
\hline Timeline for experiment & Short & Short & Long & Long & Long \\
\hline
\end{tabular}

glycocalyx remodeling and loss in experimental FSGS and in vitro (Ebefors et al., 2019). In diabetic kidney disease, GEC dysfunction and glycocalyx damage represent initiating steps in diabetic albuminuria in humans and in experimental models (Zhao et al., 2006; Satchell and Tooke, 2008; Yuen et al., 2012; Dogne et al., 2016; Lassen and Daehn, 2020).

Importantly, bidirectional signaling enables cells in the glomeruli to function effectively, where podocytes control GEC growth and survival via crosstalk of paracrine vascular endothelial growth factor alpha (VEGFA and VEGF-R; Sison et al., 2010; Jeansson et al., 2011). Crosstalk also exists between endothelial and mesangial cells (PDGF-B and PDGFR- $\beta$ ) and between podocytes and mesangial cells (CCL21 and CCR7; Vaughan and Quaggin, 2008; Schlondorff and Banas, 2009). Hence, all components contribute to the overall structure and function of this complex barrier, and model systems that can recapitulate in vivo biology and microenvironment would provide a platform for studying cell crosstalk and feedback regulation and open up the new therapeutic strategies specifically targeting the GFB.

\section{Modeling the Glomerular Filtration Barrier}

The unique environment and complex interactions between the specialized cells in the GFB make modeling glomerular disease particularly challenging. Podocytes are a key target cell for injury in the evolution of segmental sclerosis lesions of proteinuric diseases, and their morphology is critical for glomerular filtration. However, once isolated, podocytes rapidly dedifferentiate and lose their specialized morphology, making it difficult to study their function in vitro. Immortalized mouse and human podocyte cell lines have played a fundamental role in advancing podocyte research, but they lack defined foot processes as well as slit diaphragms. Efforts have been made to improve podocytes in culture to more closely recapitulate their in vivo phenotypic characteristics. By modulating the ECM, which affects most aspects of cellular behavior, researchers have established that growing primary rat podocytes in the presence of heparin and all-trans retinoic acid on laminin-coated plates resulted in podocytes with primary processes that further bifurcated and interdigitated with adjacent cells (Yaoita et al., 2018). Growing podocytes in 


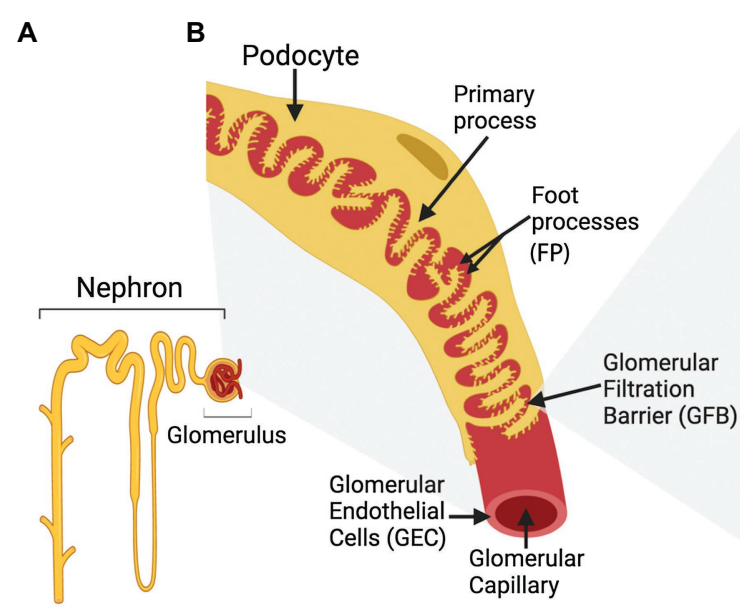

C

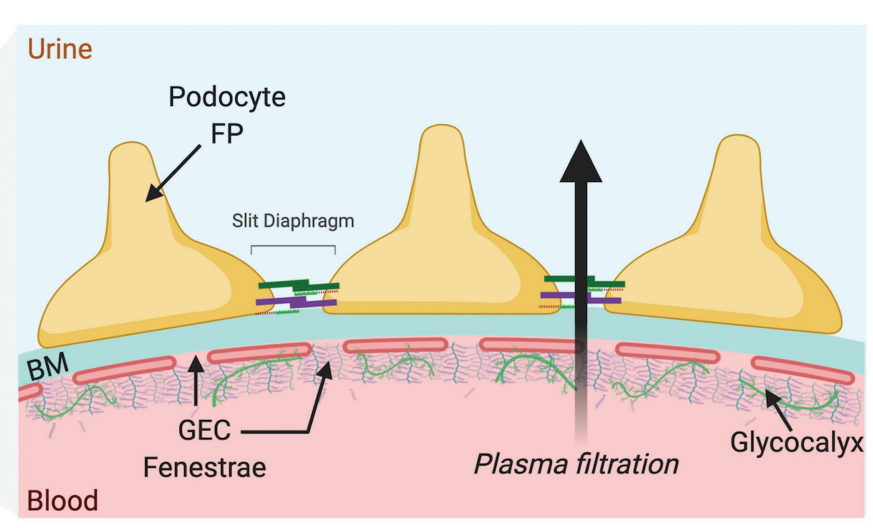

FIGURE 1 | Schematic drawing of a single nephron and glomerulus, a glomerular capillary vessel, and the glomerular filtration barrier (GFB). (A) A single nephron comprising the blood-filtering glomerulus, enveloped by Bowman's capsule that connects to the proximal tubule at the start of the urine-modifying tubular system. (B) The luminal surface of the glomerular capillary vessel is covered by glomerular endothelial cells (GECs), while podocytes wrap around the outside of the vessel with primary and foot processes (FT), forming an interdigitated pattern. Neighboring FPs are bridged by the slit diaphragm, one of the several essential components for glomerular permselectivity. The blood is filtered over this capillary barrier, and the pre-urine produced is forwarded from the Bowman's capsule into the lumen of the proximal tubule. (C) The schematic cross section of the GFB displays the GEC fenestrae attached to the basement membrane (BM) and covered luminally by glycoproteins, proteoglycans, and glycosaminoglycans of the glycocalyx, important for maintaining the charge selectivity of the GFB. On the opposite side of the BM, the podocyte FPs are attached. The FPs interlink by slit diaphragm proteins, such as nephrin and podocin, are important for the restriction of albumin by the GFB. The arrow shows the direction of plasma filtration over the barrier.

a gelatin microbial transglutaminase platform tuned to the stiffness of healthy glomeruli promoted the differentiation and maturation response of podocytes (Hu et al., 2017). Other approaches involve culturing podocytes on nanoporous surfaces with grooves. This method showed that podocytes were better differentiated, had organized actin cytoskeleton stress fibers, and developed vinculinpositive focal adhesions (Zennaro et al., 2016). Microscale curvature surfaces have also been shown to promote podocyte differentiation in vitro (Korolj et al., 2018). By growing podocytes on topographic substrates, the authors showed augmented nephrin expression and structured F-actin arrangement within cells. The curved surfaces promoted process formation with interdigitation and improved barrier function compared to podocytes grown on flat substrates (Korolj et al., 2018). Bioengineered surfaces that artificially induce branch formation have been developed by growing podocytes on a $3 \mathrm{D}$ geometry that mechanically enforces the arborization of individual podocytes (Ron et al., 2017). The formation of peripheral projections showed increased slit diaphragm proteins (nephrin, podocin, and NEPH1) and synaptopodin, as well as actinin- 4 cross-linked actin stress fibers properly localized within these peripheral processes. In addition to observing slit diaphragmlike cell-cell junctions, the authors also demonstrated that on these surfaces, podocytes had a significant increase in expression of genes related to podocyte function, hence a more mature physiological phenotype (Ron et al., 2017). The next steps are already underway involving the derivation and generation of human pluripotent stem cells into podocyte-like cells (Yaoita et al., 2018; Ge et al., 2020). These will be instrumental for future studies and high-content screening for podocentric therapies, and for integration into more complex model systems discussed below.
There are also challenges in obtaining, culturing, and maintaining GECs in vitro. GECs differ in anatomy to most other endothelial cells in the body and are defined by their fenestrations, which are important for the function of the filtration barrier (Satchell and Braet, 2009; Fogo and Kon, 2010). The fenestrations lack diaphragm but are covered with a glycocalyx. Mimicking GEC function in vitro has been challenging as they lose fenestrations in culture. This may be due to their dependence on podocytederived growth factors for their viability through intercellular crosstalk and interactions with the GBM. However, the very first human glomerular endothelial cell (GEnC) line, developed by Satchell et al. (2006), was shown to have fenestrations in response to VEGF, and over the years, it has proved to be a useful tool in GFB research, including in studies of glomerular cell interactions (Boor et al., 2010; Byron et al., 2014). The importance of VEGF-C on GEC monolayer permeability has been demonstrated through the measurement of trans-endothelial electrical resistance (TEER) as an indicator of the integrity of GEC's intercellular junctions (Ramnath and Satchell, 2020) and the passage of fluorescencelabeled BSA (Foster et al., 2008). The authors found that VEGF-C increased TEER and limited albumin passage, in contrast to the effect of VEGF-A, suggesting that these podocyte-derived growth factors regulate the permeability of GECs in the GFB (Foster et al., 2008). Although quantification of the glomerular endothelial glycocalyx in vivo has been achieved by direct labeling or indirect measurements (Hjalmarsson et al., 2004; Dane et al., 2015), measuring the glycocalyx in cultured GECs has been challenging due to the nature of this invisible layer. Recently, atomic force microscope elastography was used to successfully measure $3 \mathrm{D}$ biomechanical properties of the glycocalyx on murine GECs through direct contact by deflection of a cantilever, without 
exposing cultured cells to fixation or staining procedures that alter the fragile structure (Ebefors et al., 2019). An additional requirement of GECs function is fluid flow, which is absent in monocultures, leading to loss of the influence of shear stress on cell shape and signal transduction that is present under physiological conditions (Ballermann et al., 1998). One shear stress-inducible transcription factor is Krüppel-like factor 2 (KLF2; Lee et al., 2006), an important regulator of hemodynamic signals in endothelial cells that has been shown to be dysregulated in diabetic kidney disease. Importantly, the endothelial cell-specific knockout of KLF2 results in worsened endothelial cell and podocyte injury in an experimental model of type 1 diabetes (Zhong et al., 2014).

In addition to the challenges of providing a favorable biophysical environment for glomerular cells, ideal models of the GFB should allow for adjustment of the GFR, given that hyperfiltration occurs under physiological conditions, such as during pregnancy, and is commonly observed in $\mathrm{DKD}$, polycystic kidney disease, and sickle-cell anemia (Helal et al., 2012; Cheung and Lafayette, 2013). A physiological decline in GFR is conversely associated with advancing age (Musso and Oreopoulos, 2011). Hence, adjustable GFR is an important consideration for the physiological relevance of in vitro GFB models that can be addressed by using microfluidic devices. Innovative tools are still needed to account for tubuloglomerular feedback (TGF) that is regulated via macula densa cells in the distal tubule and the myogenic response (Vallon, 2003). TGF has mostly been studied in vivo due to the challenges of studying the intricate signaling between these cells in vitro.

Despite some of the challenges mentioned, in vitro models are making substantial progress as an alternative or complement to in vivo experimental models for mechanistic studies of the GFB components and intercellular crosstalk. In the following sections, we review the recent developments in this evolving field.

\section{STUDYING GLOMERULAR CELL CROSSTALK}

Two-dimensional (2D) cultures are a simple culture system to study glomerular cell-specific effects, as they provide screening of large numbers of conditions and treatments that would otherwise not be possible in vivo (Table 1). To study glomerular crosstalk, conditioned medium transfer is necessary when using $2 \mathrm{D}$ cultures. Despite the inherent limitations of $2 \mathrm{D}$ cultures, this system allows to chronologically separate cellular signaling events of pathogenic stimuli that ultimately lead to cell and/ or organ dysfunction.

There are different strategies used for the conditioned medium transfer, and these have been well described by Hanspal et al. in the context of amyotrophic lateral sclerosis research (Hanspal et al., 2017). The simplest strategy consists of whole medium transfer from one monoculture to another in separate culture vessels. There can also be an intermediate step of extraction or enrichment of specific media components before medium transfer to the acceptor cell culture. Insights from this approach have provided evidence for the pathologic effects of the milieu in women with preeclampsia, where factors including endothelin-1 from GECs exposed to the serum from patients with preeclampsia resulted in shedding of nephrin from podocytes cell surface via endothelin receptor A after media transfer (Collino et al., 2008). Another study utilized the transfer of purified exosomes from high glucose-treated GECs to podocytes and found that TGF $\beta$ mRNA, carried by the extracellular vesicles, contributed to podocyte dedifferentiation epithelialmesenchymal transition (Wu et al., 2017). The authors found the same mechanism of exosomes containing TGF $\beta$ mRNA to contribute to mesangial cell proliferation and matrix production through a similar experimental setup, as well as through tailvain injections of the purified exosomes from high glucosetreated GECs in C57BL/6 mice (Wu et al., 2016). Furthermore, the studies of TGF $\beta$-containing exosomes by another group supported the involvement of these extracellular vesicles in glomerular crosstalk following high glucose stimulation (Wang et al., 2018b). Exosomes have emerged as a novel vector for cell-cell communication in the kidney, and they are beginning to be recognized more and more as a critical player in the pathogenesis of kidney disease and decline in renal function.

Co-culture of two or more cell types offers increased complexity over monocultures when studying glomerular crosstalk. Open microfluidics systems allow simultaneous paracrine signaling between two separated cell populations by sharing culture medium and hence allow for exchange of soluble factors and transient signals (Zhang et al., 2020). In transwell systems, two distinct cell types are separated by a porous membrane (Hanspal et al., 2017), where a bidirectional exchange of signaling molecules can occur with or without direct cell-cell contact (Table 1). Li and colleagues demonstrated the applicability of their co-culture model of the GFB for studies of drug testing and intracellular signaling, using murine podocytes and GECs on opposite sides of a collagen IV-coated polyethylene terephthalate membrane ( $\mathrm{Li}$ et al., 2016). More recently, the same research group successfully exchanged the murine glomerular cells for human immortalized GECs and podocytes, and reported an increase in albumin leak after exposure to sera from patients with recurrent FSGS, compared to genetic or non-recurrent forms (Li et al., 2020b). Casalena et al. have demonstrated that both high glucose and serum from diabetic mice susceptible to developing diabetic kidney disease disrupt mitochondrial function and cause oxidative stress in GECs. Interestingly, the transfer of factors released by the stressed GECs mediated podocyte cell death in transwell co-cultures, as well as in media exchange (Casalena et al., 2020). Given that bi-directional communication can still occur while cells are physically separated, this approach allows for subsequent interrogation of cell-specific responses. This approach has also been used to define podocyteto-GEC-to-podocyte crosstalk in the pathogenesis of FSGS by shedding light on the role molecules, such as endothelin-1/ endothelin receptor type A-mediated glomerular endothelial cell dysfunction, which was shown to be required for podocyte depletion and progression of glomerulosclerosis (Daehn et al., 2014).

Exposure of GECs to laminar shear forces found in vivo adds physiological relevance to the transwell co-culture model of the GFB. Studies by Slater et al. used both conditioned medium transfer and co-culture of human GECs and podocytes to investigate how ERK5 activation and KLF2 transcription 
(associated with endothelial cell shear stress in large vessels) affected the glomerular microvasculature (Slater et al., 2012). Their findings demonstrated the existence of intercellular signaling from GECs exposed to chronic laminar shear stress that affects podocytes. In another study by the same research group, GECs and podocytes were co-cultured on opposite sides of a polycaprolactone/electrospun collagen membrane to closer mimic the GBM, which was shown to enable cell-cell contact (Slater et al., 2011). Differences in between the conditioned medium transfer and the co-culture settings suggest that spatial separation between crosstalking cell types is an important consideration.

The models described so far provide robust high-throughput, high-content reductionist assay systems. They have provided a wealth of information on the fundamental biological and disease processes of the GFB. Nevertheless, they provide a limited physiological context of the filtration barrier. Since there is growing awareness of the interconnections between cells and the ECM surrounding them, there is substantial effort by the community to develop model systems that can better reflect the complex microenvironment cells encounter in a tissue.

\section{D Culture Models of the GFB}

Organs-on-a-chip have been developed for complex organs such as liver (Beckwitt et al., 2018), heart (Agarwal et al., 2013), gut (Kim et al., 2012; Kim and Ingber, 2013), lungs (Huh et al., 2010, 2012), and brain (Moreno et al., 2015). The goal has not been to mimic the whole organs, but rather to study complex parts of an organ in a more physiological context. In the renal field, chips for modeling the proximal tubules (Jang et al., 2013; Hoppensack et al., 2014; Wilmer et al., 2016) as well as the filtration barrier are being developed. An ideal model of the GFB would include cell-to-cell and cellto-ECM interactions, biomimetic micromechanical properties, shear flow, oxygen and nutrient/waste exchange, and a functional permselective filtration barrier. In the last decade, the development of microfluidic platforms that allow co-culture of cells under flow (Bhatia and Ingber, 2014) and stretch (Huh et al., 2013) has emerged (Table 1) and these continue to evolve. Here, we describe some examples.

To study the effect of hypertension on the filtration barrier, Zhou et al. developed a glomerulus-on-a-chip using murine immortalized GECs and podocytes. The cells were separated in the chip by a polycarbonate membrane coated with basement membrane extracts, and the authors increased the flow in the upper channel of the chip harboring the GECs (Zhou et al., 2016). Increasing the mechanical force led to cell damage, loss of junctions, and changes to the cell's cytoskeleton, leading to increased leakage (Zhou et al., 2016). In an in vitro model of diabetic kidney disease, Wang et al. developed a glomeruluson-a-chip using glomeruli isolated from rats. The chip consisted of five channels, a capillary in the middle and collection channels on the outside, with the channels in between filled with gel. Isolated glomeruli were injected in the capillary channel and allowed to attach for the cells to spread and form a barrier under flow. GECs and podocytes were identified by CD31 and synaptopodin staining, respectively. High glucose treatment enhanced the permeability to proteins and increased reactive oxygen species production and podocyte detachment (Wang et al., 2017). Musah et al. developed a glomerulus-on-a-chip with fluidics and strain by using vacuum channels on the side of the channel harboring the GECs and podocytes (Musah et al., 2017). The authors developed podocytes derived from human induced pluripotent stem cells (iPSCs) and used them in combination with human GECs separated by a porous polydimethylsiloxane membrane coated with laminin. The mechanical strain was shown to increase the expression of nephrin and secretion of VEGF-A by the podocytes. Albuminuria and podocyte damage were observed with adriamycin treatment, underscoring the resemblance to the in vivo setting (Musah et al., 2017). These models however lack GBM; hence, Petrosyan et al. developed a glomerulus-on-a-chip without an artificial membrane between GECs and podocytes (Petrosyan et al., 2019). The authors allowed both cell types to interact and to generate a layer of ECM components. Human GECs and podocytes were obtained from the same donor; cells were separated by collagen I and eventually formed a basement membrane between the cell layers. GECs were further shown to develop a glycocalyx layer. The cells could be maintained in the chip for at least a month, enabling long-term experiments. Exposure of chips to puromycin aminonucleoside induced podocyte injury and loss of permselectivity for albumin. Adding serum from patients with membranous nephropathy (MN) resulted in albumin leakage, which was prevented by treatment with $\alpha$-MSH. Using podocytes derived from a patient with Alport syndrome rendered improper filtration, supporting the chips potential for the use in personalized medicine (Petrosyan et al., 2019).

Given that the glomerulus in situ has a complex structure with intricate microvascular capillary networks in a unique geometry that could play a role in the development and function of podocytes (Falkenberg et al., 2017), there have been significant efforts to generate $3 \mathrm{D}$ models with complex microvascular networks using 3D bioprinting technology. Rayner et al. demonstrated the use of a multiphoton microscopy-guided $3 \mathrm{D}$ printing technique to generate perfusable vascular networks with diameters as small as $10 \mu \mathrm{m}$ (Rayner et al., 2021). They further demonstrate bioprinting of a glomerular-like microvascular network that supports endothelial lumen formation; however, they still require the incorporation of podocytes and mesangial cells to recapitulate the glomerular physiology and to study cell-cell crosstalk. Other developments include the glomerulus-on-a-plate, recently developed by using a microfluidic topographical hollow fiber (Xie et al., 2020). This system uses a tubular-like perfusable channel to seed GECs in a glomerulus-like knot with microconvex topography, filled with hydrogel and covered with murine podocytes. The fibers were mounted in specialized 96-well plates with inlet and outlet wells allowing flow to be applied by either gravity or syringe pump. Perfusing the lumen with albumin showed no leakage of over the barrier, while small molecules could readily pass. However, adriamycin treatment was shown to increase the passage of BSA over the barrier, but only mildly damaged podocytes (Xie et al., 2020). 
Current GFB 3D culture model technologies have a number of drawbacks, such as recirculating instead of a continuous flow, long culture times to achieve fully confluent layers, lack of a basement membrane, and limited throughput. However, these models still hold great promise for improving our understanding of glomerular crosstalk and their potential use for personalized and precision medicine. In the future, chips where cells can form a basement membrane without separating gels or man-made membranes will emerge, and the inclusion of mesangial cells, pericytes, and parietal epithelial cells to the chips would enable all the intricate signaling which takes place in the glomerulus.

\section{Scaffold-Free 3D Cultures}

Scaffold-free 3D cultures are anchorage-independent models that rely on the self-aggregation of cells in specialized culture plates with ultra-low attachment coating that promotes spheroid formation. Multicellular spheroids have been shown to recapitulate physiological characteristics of tissues and tumors with regard to cell-cell contact, and allow for natural cell-ECM interactions (Sutherland, 1988). Glomeruloid spheres have been developed using human mesenchymal stem cells, HUVECs, and HEKs (Abe et al., 2019). These spheroids expressed several podocyte markers and were stable for at least 5 days. Adding serum from patients with FSGS resulted in the collapse of the spheres (Abe et al,, 2019). In 2020, Cho et al. demonstrated a novel pressure-assisted network for droplet accumulation method for high-throughput generation of uniform microtissues. As a proof of principle, they generated glomerulus-like microtissues using immortalized mouse podocytes and mesenchymal stem cells (Cho et al., 2020). More recently, Sobreiro-Almeida et al. observed that the addition of retinoic acid to an organotypic model of human renal progenitor cells resulted in spheroids with a preferential glomerular differentiation. Using a hanging drop culture technique to form spheroids, they showed that these spheroids remain viable over a period of 28 days and display an elevated expression of PAX2 and NPHS1 in the presence of retinoic acid. Further, co-culture with microvascular endothelial cells resulted in more compact organization of the spheroids (Sobreiro-Almeida et al., 2021).

These scaffold-free 3D cultures are not barrier models, and many questions remain: in particular, about the composition of the spheres. And improvement in oxygenation through integration of endothelial cells has not been examined in this setting. Today's glomeruloid spheres can provide insights for podocyte-ECM interactions and can be adapted to medium- or high-throughput screening assays. There is still the need for culture optimization to enhance reproducibility of spheroids in culture and to study GFB components, while maintaining a small enough size for sufficient nutrient exchange. However, this area of research is moving fast, and we will undoubtedly see advances in the years to come.

\section{Organoids}

Attempts to fully culture organs in vitro have led to the development of organoids, self-organized 3D aggregations of cells. Over the last few years, these developments have provided researchers the opportunity to establish near-physiological models to study human development and diseases. Organoids can be derived from embryonic stem cells or iPSCs. The kidney is an anatomically complex organ with numerous different cell types, which makes it difficult to get organoids containing all renal structures including a functional filtration barrier. As of today, organoids are premature, and as such, they do not represent ideal modeling systems for studies of the GFB; however, they hold promise to be so in the future.

Embryonic kidneys are divided into the metanephric mesenchyme and the ureteric bud. Nephron progenitor cells in the metanephric mesenchyme are the origin of the glomeruli, Bowman's capsule, and the renal tubules, and stromal progenitor cells give rise to interstitial cells. The ureteric bud is the origin of the collecting ducts. During development, intricate signaling leads to differentiation of cells and the formation of a mature kidney. In order to form kidney organoids, this signaling needs to be applied to embryonic or pluripotent stem cells. With this in mind, the development of differentiation protocols for embryonic and iPSCs toward renal cells (Xia et al., 2013; Taguchi et al., 2014; Takasato et al., 2014) was rapidly followed by the first reports of kidney organoids (Morizane et al., 2015; Takasato et al., 2015). Kidney organoids have been characterized via single-cell sequencing and have been found to contain developing podocytes, parietal epithelial cells, tubular cells, collecting ducts, and interstitial and stromal cells. Missing or underrepresented cells with current methods are GECs, mesangial cells, principal and intercalated cells (Czerniecki et al., 2018; Wu et al., 2018; Combes et al., 2019), and immune cells. Although glomerulus-like structures are formed, they mainly consist of early podocytes, and these have the potential to be explored further to study podocytopathies (Sharmin et al., 2016; Kim et al., 2017; Hale et al., 2018). Hale et al. describe a protocol for kidney organoids from iPSCs and compared the expression to human immortalized podocyte cell lines. Podocytes derived from organoids were shown to have an improved expression profile, as well as a GBM (Hale et al., 2018). Genetic modifications targeting podocytes have also been used in kidney organoids to explore congenital nephrotic syndrome (Kim et al., 2017; Hale et al., 2018; Tanigawa et al., 2018). In addition, to better study the GFB, improvements in methods that promote maturation and vascularization of the organoids have been reported recently, such as culturing kidney organoids on millifluidic chips (Homan et al., 2019), or transplantation of human kidney organoids into the subcapsular of mouse kidneys (van den Berg et al., 2018). In the latter, the authors demonstrated an improvement in the formation of a GBM with the development of a fenestrated endothelium in glomeruli (van den Berg et al., 2018). By modulating biophysical cues, such as ECM stiffness, Garreta et al. were able to accelerate kidney organoid generation from iPSCs (Garreta et al., 2019). They showed that implantation of kidney organoids into chick chorioallantoic membrane (CAM) resulted in vascularization of the organoids within 5 days. They further generated soft hydrogels that display similar mechanical properties as CAM to study if soft substrates drive kidney organoid generation compared to stiffer substrates. They observed that soft matrix environment resulted in kidney organoids that display similar protein expression as a fetal human kidney. Although the kidney organoids still are embryonic in development and need an in vivo environment for vascularization, 
further characterization of the role of substrate stiffness can improve kidney organoid differentiation. Another limitation of the current organoid systems is the heterogeneity and batch-to-batch variation during initial formation and maturation. To address this, Dr. Little's group have employed two different approaches for scaling up the generation of kidney organoids with less heterogeneity and higher reproducibility. Kumar et al. demonstrated a method to scale up the generation of kidney micro-organoids in suspension culture (Kumar et al., 2019). Using this method, they were able to generate 8,000-10,000 kidney micro-organoids in an even size range. These organoids are less than $200-300 \mu \mathrm{m}$ in final size, much smaller compared to standard organoids, which allows efficient nutrient diffusion to the core of the organoids. However, they showed limited utility with respect to extended long-term cultures due to the absence of vascularization. Lawlor et al. employed extrusion bioprinting method to plate cell aggregates that mature into kidney organoids, which partially eliminates organoid heterogeneity and enables scaling up of throughput (Lawlor et al., 2021). Using this technique, they were able to generate 200 organoids in $10 \mathrm{~min}$. In addition to reducing variability, extrusion bioprinting can also be used to alter the conformation of the organoids, to generate a spheroid or a rectangular cell aggregate patch based on the extruding tip movement. The authors observe that the rectangular conformation yielded a greater number of nephron units compared to the spheroid conformation (Lawlor et al., 2021), which with further improvements may be useful for the development of transplantable kidney tissues.

Despite the many challenges that still remain for organoids to fully resemble mature human kidneys, including less off targets cells as described in detail in the review by Geuens et al. (2020), organoid biobanks as repository for drug screening and development are emerging (Calandrini et al., 2020) and have the potential for applications in precision medicine.

\section{FUTURE PERSPECTIVE}

The lack of specific treatments for diseases of the GFB is a worldwide health issue. The need for new explorative in vitro models is paramount to elucidate the intricate signaling of cells in the GFB. Today, there is greater recognition that components of the GFB work as an integrated functional unit. As more and more new tools become available, such as iPSCs in culture and 3D model systems, we shall look to integrate these human-relevant in vitro models with data-driven and mechanistic modeling as well as artificial intelligence-driven methods that can assist with in silico drug discovery and modeling (Azeloglu et al., 2014),

\section{REFERENCES}

Abe, H., Sakurai, A., and Ochi, A. (2019). Induction of steady-state glomeruloid sphere by self-assembly from human embryonic kidney cells. Biochem. Biophys. Res. Commun. 508, 654-659. doi: 10.1016/j.bbrc.2018.11.160

Agarwal, A., Goss, J. A., Cho, A., McCain, M. L., and Parker, K. K. (2013). Microfluidic heart on a chip for higher throughput pharmacological studies. Lab Chip 13, 3599-3608. doi: 10.1039/c3lc50350j which will inevitably streamline time-consuming and costly experiments. As we gain our understanding on other aspects that influence GFB function, such as tubuloglomerular crosstalk (Tasnim and Zink, 2012; Wang et al., 2018a), opportunities to "plug-in" modules will provide insights from the whole nephron's perspective and even distant organ crosstalk. Together with the increasingly quantitative precision medicine approaches that can collate and combine clinical data with genomic information, these joint efforts can help guide the design of novel drug candidates and move the field toward the common goal of treating patients with better therapies for diseases of the GFB.

\section{CONCLUSION}

As these experimental model systems continue to evolve and improve in terms of their physiological context and throughput, model systems have a huge potential to help unravel the molecular mechanisms of GFB breakdown and the pathogenic crosstalk signaling that may drive disease. These developments should minimize the use of animal models and accelerate discoveries by enabling the platforms for personalized and precision medicine to lower drug-induced adverse events, and identify new targets for treatments of kidney diseases that affect the filtration barrier.

\section{AUTHOR CONTRIBUTIONS}

Conceptualization by KE, EL and ID. KE, EL, NA, EA and ID wrote the manuscript. All authors contributed to the article and approved the submitted version.

\section{FUNDING}

ID is supported by the National Institutes of Health grant R01DK097253. ID and EA are supported by the Department of Defense CDMRP grants W81XWH-20-1-0836 (ID) and W81XWH-20-1-0837 (EA).

\section{ACKNOWLEDGMENTS}

We acknowledge all front-line workers for all the sacrifices that you and your family are making to help us all get through these tough times dealing with the global pandemic. Words are not enough to thank you for your strength, courage, and dedication. Illustrations were created using BioRender.

Anandakrishnan, N., and Azeloglu, E. U. (2020). Kidney tissue engineering for precision medicine. Nat. Rev. Nephrol. 16, 623-624. doi: 10.1038/ s41581-020-00355-6

Azeloglu, E. U., Hardy, S. V., Eungdamrong, N. J., Chen, Y., Jayaraman, G., Chuang, P. Y., et al. (2014). Interconnected network motifs control podocyte morphology and kidney function. Sci. Signal. 7:ra12. doi: 10.1126/scisignal.2004621

Ballermann, B. J. (2007). Contribution of the endothelium to the glomerular permselectivity barrier in health and disease. Nephron Physiol. 106, 19-25. doi: $10.1159 / 000101796$ 
Ballermann, B. J., Dardik, A., Eng, E., and Liu, A. (1998). Shear stress and the endothelium. Kidney Int. Suppl. 67, S100-S108. doi: 10.1046/ j.1523-1755.1998.06720.x

Bao, Y.-W., Yuan, Y., Chen, J.-H., and Lin, W.-Q. (2018). Kidney disease models: tools to identify mechanisms and potential therapeutic targets. Zool. Res. 39, 72-86. doi: 10.24272/j.issn.2095-8137.2017.055

Becker, G. J., and Hewitson, T. D. (2013). Animal models of chronic kidney disease: useful but not perfect. Nephrol. Dial. Transplant. 28, 2432-2438. doi: $10.1093 / \mathrm{ndt} / \mathrm{gft} 071$

Beckwitt, C. H., Clark, A. M., Wheeler, S., Taylor, D. L., Stolz, D. B., Griffith, L., et al. (2018). Liver 'organ on a chip'. Exp. Cell Res. 363, 15-25. doi: 10.1016/j. yexcr.2017.12.023

Bhatia, S. N., and Ingber, D. E. (2014). Microfluidic organs-on-chips. Nat. Biotechnol. 32, 760-772. doi: 10.1038/nbt.2989

Boels, M. G., Avramut, M. C., Koudijs, A., Dane, M. J., Lee, D. H., van der Vlag, J., et al. (2016). Atrasentan reduces albuminuria by restoring the glomerular endothelial glycocalyx barrier in diabetic nephropathy. Diabetes 65, 2429-2439. doi: 10.2337/db15-1413

Boor, P., van Roeyen, C. R., Kunter, U., Villa, L., Bucher, E., Hohenstein, B., et al. (2010). PDGF-C mediates glomerular capillary repair. Am. J. Pathol. 177, 58-69. doi: 10.2353/ajpath.2010.091008

Boreström, C., Jonebring, A., Guo, J., Palmgren, H., Cederblad, L., Forslöw, A., et al. (2018). A CRISP(e)R view on kidney organoids allows generation of an induced pluripotent stem cell-derived kidney model for drug discovery. Kidney Int. 94, 1099-1110. doi: 10.1016/j.kint.2018.05.003

Boute, N., Gribouval, O., Roselli, S., Benessy, F., Lee, H., Fuchshuber, A., et al. (2000). NPHS2, encoding the glomerular protein podocin, is mutated in autosomal recessive steroid-resistant nephrotic syndrome. Nat. Genet. 24, 349-354. doi: 10.1038/74166

Brenner, B. M., Hostetter, T. H., and Humes, H. D. (1978). Molecular basis of proteinuria of glomerular origin. N. Engl. J. Med. 298, 826-833. doi: 10.1056/NEJM197804132981507

Byron, A., Randles, M. J., Humphries, J. D., Mironov, A., Hamidi, H., Harris, S., et al. (2014). Glomerular cell cross-talk influences composition and assembly of extracellular matrix. J. Am. Soc. Nephrol. 25, 953-966. doi: 10.1681/ ASN.2013070795

Calandrini, C., Schutgens, F., Oka, R., Margaritis, T., Candelli, T., Mathijsen, L., et al. (2020). An organoid biobank for childhood kidney cancers that captures disease and tissue heterogeneity. Nat. Commun. 11:1310. doi: 10.1038/ s41467-020-15155-6

Casalena, G. A., Yu, L., Gil, R., Rodriguez, S., Sosa, S., Janssen, W., et al. (2020). The diabetic microenvironment causes mitochondrial oxidative stress in glomerular endothelial cells and pathological crosstalk with podocytes. Cell Commun. Signal 18:105. doi: 10.1186/s12964-020-00605-x

Cheung, K. L., and Lafayette, R. A. (2013). Renal physiology of pregnancy. Adv. Chronic Kidney Dis. 20, 209-214. doi: 10.1053/j.ackd.2013.01.012

Chew, C., and Lennon, R. (2018). Basement membrane defects in genetic kidney diseases. Front. Pediatr. 6:11. doi: 10.3389/fped.2018.00011

Cho, C. Y., Chiang, T. H., Hsieh, L. H., Yang, W. Y., Hsu, H. H., Yeh, C. K., et al. (2020). Development of a novel hanging drop platform for engineering controllable 3D microenvironments. Front. Cell Dev. Biol. 8:327. doi: 10.3389/ fcell.2020.00327

Collino, F., Bussolati, B., Gerbaudo, E., Marozio, L., Pelissetto, S., Benedetto, C., et al. (2008). Preeclamptic sera induce nephrin shedding from podocytes through endothelin-1 release by endothelial glomerular cells. Am. J. Physiol. Renal Physiol. 294, F1185-F1194. doi: 10.1152/ajprenal.00442.2007

Combes, A. N., Zappia, L., Er, P. X., Oshlack, A., and Little, M. H. (2019). Single-cell analysis reveals congruence between kidney organoids and human fetal kidney. Genome Med. 11:3. doi: 10.1186/s13073-019-0615-0

Czerniecki, S. M., Cruz, N. M., Harder, J. L., Menon, R., Annis, J., Otto, E. A., et al. (2018). High-throughput screening enhances kidney organoid differentiation from human pluripotent stem cells and enables automated multidimensional phenotyping. Cell Stem Cell 22, 929.e4-940.e4. doi: 10.1016/j. stem.2018.04.022

Daehn, I., Casalena, G., Zhang, T., Shi, S., Fenninger, F., Barasch, N., et al. (2014). Endothelial mitochondrial oxidative stress determines podocyte depletion in segmental glomerulosclerosis. J. Clin. Invest. 124, 1608-1621. doi: $10.1172 /$ JCI71195
Daehn, I. S. (2018). Glomerular endothelial cells stress and cross-talk with podocytes in the development of diabetic kidney disease. Front. Med. 5:76. doi: $10.3389 /$ fmed.2018.00076

Dane, M. J., van den Berg, B. M., Lee, D. H., Boels, M. G., Tiemeier, G. L., Avramut, M. C., et al. (2015). A microscopic view on the renal endothelial glycocalyx. Am. J. Physiol. Renal Physiol. 308, F956-F966. doi: 10.1152/ ajprenal.00532.2014

Dogne, S., Rath, G., Jouret, F., Caron, N., Dessy, C., and Flamion, B. (2016). Hyaluronidase 1 deficiency preserves endothelial function and glycocalyx integrity in early streptozotocin-induced diabetes. Diabetes 65, 2742-2753. doi: $10.2337 / \mathrm{db} 15-1662$

Ebefors, K., Wiener, R. J., Yu, L., Azeloglu, E. U., Yi, Z., Jia, F., et al. (2019). Endothelin receptor-A mediates degradation of the glomerular endothelial surface layer via pathologic crosstalk between activated podocytes and glomerular endothelial cells. Kidney Int. 96, 957-970. doi: 10.1016/j. kint.2019.05.007

Falkenberg, C. V., Azeloglu, E. U., Stothers, M., Deerinck, T. J., Chen, Y., He, J. C., et al. (2017). Fragility of foot process morphology in kidney podocytes arises from chaotic spatial propagation of cytoskeletal instability. PLoS Comput. Biol. 13:e1005433. doi: 10.1371/journal. pcbi. 1005433

Fogo, A. B., and Kon, V. (2010). The glomerulus-a view from the inside-the endothelial cell. Int. J. Biochem. Cell Biol. 42, 1388-1397. doi: 10.1016/j. biocel.2010.05.015

Foster, R. R., Slater, S. C., Seckley, J., Kerjaschki, D., Bates, D. O., Mathieson, P. W., et al. (2008). Vascular endothelial growth factor-C, a potential paracrine regulator of glomerular permeability, increases glomerular endothelial cell monolayer integrity and intracellular calcium. Am. J. Pathol. 173, 938-948. doi: 10.2353/ajpath.2008.070416

Garreta, E., Prado, P., Tarantino, C., Oria, R., Fanlo, L., Marti, E., et al. (2019). Fine tuning the extracellular environment accelerates the derivation of kidney organoids from human pluripotent stem cells. Nat. Mater. 18, 397-405. doi: 10.1038/s41563-019-0287-6

Ge, X., Zhang, T., Yu, X., Muwonge, A. N., Anandakrishnan, N., Wong, N. J., et al. (2020). LIM-nebulette reinforces podocyte structural integrity by linking actin and vimentin filaments. J. Am. Soc. Nephrol. 31, 2372-2391. doi: 10.1681/ASN.2019121261

Geuens, T., van Blitterswijk, C. A., and LaPointe, V. L. S. (2020). Overcoming kidney organoid challenges for regenerative medicine. NPJ Regener. Med. 5:8. doi: 10.1038/s41536-020-0093-4

Hackl, M. J., Burford, J. L., Villanueva, K., Lam, L., Susztak, K., Schermer, B., et al. (2013). Tracking the fate of glomerular epithelial cells in vivo using serial multiphoton imaging in new mouse models with fluorescent lineage tags. Nat. Med. 19, 1661-1666. doi: 10.1038/nm.3405

Hale, L. J., Howden, S. E., Phipson, B., Lonsdale, A., Er, P. X., Ghobrial, I., et al. (2018). 3D organoid-derived human glomeruli for personalised podocyte disease modelling and drug screening. Nat. Commun. 9:5167. doi: 10.1038/ s41467-018-07594-Z

Hansen, K. U. I., Siegerist, F., Daniel, S., Schindler, M., Iervolino, A., Blumenthal, A., et al. (2020). Prolonged podocyte depletion in larval zebrafish resembles mammalian focal and segmental glomerulosclerosis. FASEB J. 34, 15961-15974. doi: 10.1096/f. 202000724R

Hanspal, M. A., Dobson, C. M., Yerbury, J. J., and Kumita, J. R. (2017). The relevance of contact-independent cell-to-cell transfer of TDP-43 and SOD1 in amyotrophic lateral sclerosis. Biochim. Biophys. Acta Mol. Basis Dis. 1863, 2762-2771. doi: 10.1016/j.bbadis.2017.07.007

Haraldsson, B., and Nystrom, J. (2012). The glomerular endothelium: new insights on function and structure. Curr. Opin. Nephrol. Hypertens. 21, 258-263. doi: 10.1097/MNH.0b013e3283522e7a

Haraldsson, B., Nyström, J., and Deen, W. M. (2008). Properties of the glomerular barrier and mechanisms of proteinuria. Physiol. Rev. 88, 451-487. doi: 10.1152/physrev.00055.2006

Helal, I., Fick-Brosnahan, G. M., Reed-Gitomer, B., and Schrier, R. W. (2012). Glomerular hyperfiltration: definitions, mechanisms and clinical implications. Nat. Rev. Nephrol. 8, 293-300. doi: 10.1038/nrneph.2012.19

Hjalmarsson, C., Johansson, B. R., and Haraldsson, B. (2004). Electron microscopic evaluation of the endothelial surface layer of glomerular capillaries. Microvasc. Res. 67, 9-17. doi: 10.1016/j.mvr.2003.10.001 
Homan, K. A., Gupta, N., Kroll, K. T., Kolesky, D. B., Skylar-Scott, M., Miyoshi, T., et al. (2019). Flow-enhanced vascularization and maturation of kidney organoids in vitro. Nat. Methods 16, 255-262. doi: 10.1038/s41592-019-0325-y

Honeycutt, A. A., Segel, J. E., Zhuo, X., Hoerger, T. J., Imai, K., and Williams, D. (2013). Medical costs of CKD in the Medicare population. J. Am. Soc. Nephrol. 24, 1478-1483. doi: 10.1681/ASN.2012040392

Hoppensack, A., Kazanecki, C. C., Colter, D., Gosiewska, A., Schanz, J., Walles, H., et al. (2014). A human in vitro model that mimics the renal proximal tubule. Tissue Eng. Part C Methods 20, 599-609. doi: 10.1089/ten.tec.2013.0446

Hu, M., Azeloglu, E. U., Ron, A., Tran-Ba, K. H., Calizo, R. C., Tavassoly, I., et al. (2017). A biomimetic gelatin-based platform elicits a pro-differentiation effect on podocytes through mechanotransduction. Sci. Rep. 7:43934. doi: 10.1038/srep43934

Huh, D., Kim, H. J., Fraser, J. P., Shea, D. E., Khan, M., Bahinski, A., et al. (2013). Microfabrication of human organs-on-chips. Nat. Protoc. 8, 2135-2157. doi: $10.1038 /$ nprot.2013.137

Huh, D., Leslie, D. C., Matthews, B. D., Fraser, J. P., Jurek, S., Hamilton, G. A., et al. (2012). A human disease model of drug toxicity-induced pulmonary edema in a lung-on-a-chip microdevice. Sci. Transl. Med. 4:159ra147. doi: 10.1126/scitranslmed.3004249

Huh, D., Matthews, B. D., Mammoto, A., Montoya-Zavala, M., Hsin, H. Y., and Ingber, D. E. (2010). Reconstituting organ-level lung functions on a chip. Science 328, 1662-1668. doi: 10.1126/science.1188302

Jang, K. J., Mehr, A. P., Hamilton, G. A., McPartlin, L. A., Chung, S., Suh, K. Y., et al. (2013). Human kidney proximal tubule-on-a-chip for drug transport and nephrotoxicity assessment. Integr. Biol. 5, 1119-1129. doi: 10.1039/ c3ib40049b

Jeansson, M., Bjorck, K., Tenstad, O., and Haraldsson, B. (2009). Adriamycin alters glomerular endothelium to induce proteinuria. J. Am. Soc. Nephrol. 20, 114-122. doi: 10.1681/ASN.2007111205

Jeansson, M., Gawlik, A., Anderson, G., Li, C., Kerjaschki, D., Henkelman, M., et al. (2011). Angiopoietin-1 is essential in mouse vasculature during development and in response to injury. J. Clin. Invest. 121, 2278-2289. doi: 10.1172/JCI46322

Kashtan, C. E. (1999). Alport syndrome: an inherited disorder of renal, ocular, and cochlear basement membranes. Medicine 78, 338-360. doi: 10.1097/00005792-199909000-00005

Kestila, M., Lenkkeri, U., Mannikko, M., Lamerdin, J., McCready, P., Putaala, H., et al. (1998). Positionally cloned gene for a novel glomerular proteinnephrin-is mutated in congenital nephrotic syndrome. Mol. Cell 1, 575-582. doi: 10.1016/S1097-2765(00)80057-X

Khramova, A., Boi, R., Fridén, V., Granqvist, A. B., Nilsson, U., Tenstad, O., et al. (2021). Proteoglycans contribute to the functional integrity of the glomerular endothelial cell surface layer and are regulated in diabetic kidney disease. Sci. Rep. 11:8487. doi: 10.1038/s41598-021-87753-3

Kim, H. J., Huh, D., Hamilton, G., and Ingber, D. E. (2012). Human gut-ona-chip inhabited by microbial flora that experiences intestinal peristalsis-like motions and flow. Lab Chip 12, 2165-2174. doi: 10.1039/c2lc40074j

Kim, H. J., and Ingber, D. E. (2013). Gut-on-a-Chip microenvironment induces human intestinal cells to undergo villus differentiation. Integr. Biol. 5, 1130-1140. doi: 10.1039/c3ib40126j

Kim, Y. K., Refaeli, I., Brooks, C. R., Jing, P., Gulieva, R. E., Hughes, M. R., et al. (2017). Gene-edited human kidney organoids reveal mechanisms of disease in podocyte development. Stem Cells 35, 2366-2378. doi: 10.1002/ stem. 2707

Koning, M., van den Berg, C. W., and Rabelink, T. J. (2020). Stem cell-derived kidney organoids: engineering the vasculature. Cell. Mol. Life Sci. 77, 2257-2273. doi: 10.1007/s00018-019-03401-0

Korolj, A., Laschinger, C., James, C., Hu, E., Velikonja, C., Smith, N., et al. (2018). Curvature facilitates podocyte culture in a biomimetic platform. Lab Chip 18, 3112-3128. doi: 10.1039/C8LC00495A

Kumar, S. V., Er, P. X., Lawlor, K. T., Motazedian, A., Scurr, M., Ghobrial, I., et al. (2019). Kidney micro-organoids in suspension culture as a scalable source of human pluripotent stem cell-derived kidney cells. Development 146:dev172361. doi: 10.1242/dev.172361

Lassen, E., and Daehn, I. S. (2020). Molecular mechanisms in early diabetic kidney disease: glomerular endothelial cell dysfunction. Int. J. Mol. Sci. 21:9456. doi: $10.3390 /$ ijms21249456
Lawlor, K. T., Vanslambrouck, J. M., Higgins, J. W., Chambon, A., Bishard, K., Arndt, D., et al. (2021). Cellular extrusion bioprinting improves kidney organoid reproducibility and conformation. Nat. Mater. 20, 260-271. doi: 10.1038/s41563-020-00853-9

Lee, J. S., Yu, Q., Shin, J. T., Sebzda, E., Bertozzi, C., Chen, M., et al. (2006). Klf2 is an essential regulator of vascular hemodynamic forces in vivo. Dev. Cell 11, 845-857. doi: 10.1016/j.devcel.2006.09.006

Li, A. S., Ingham, J. F., and Lennon, R. (2020a). Genetic disorders of the glomerular filtration barrier. Clin. J. Am. Soc. Nephrol. 15, 1818-1828. doi: 10.2215/CJN.11440919

Li, M., Alfieri, C. M., Morello, W., Cellesi, F., Armelloni, S., Mattinzoli, D., et al. (2020b). Assessment of increased glomerular permeability associated with recurrent focal segmental glomerulosclerosis using an in vitro model of the glomerular filtration barrier. J. Nephrol. 33, 747-755. doi: 10.1007/ s40620-019-00683-2

Li, M., Corbelli, A., Watanabe, S., Armelloni, S., Ikehata, M., Parazzi, V., et al. (2016). Three-dimensional podocyte-endothelial cell co-cultures: assembly, validation, and application to drug testing and intercellular signaling studies. Eur. J. Pharm. Sci. 86, 1-12. doi: 10.1016/j.ejps.2016.02.013

Little, M. H., and Takasato, M. (2015). Generating a self-organizing kidney from pluripotent cells. Curr. Opin. Organ Transplant. 20, 178-186. doi 10.1097/MOT.0000000000000174

Meyrier, A. (2011). Focal and segmental glomerulosclerosis: multiple pathways are involved. Semin. Nephrol. 31, 326-332. doi: 10.1016/j.semnephrol. 2011.06.003

Moreno, E. L., Hachi, S., Hemmer, K., Trietsch, S. J., Baumuratov, A. S., Hankemeier, T., et al. (2015). Differentiation of neuroepithelial stem cells into functional dopaminergic neurons in 3D microfluidic cell culture. Lab Chip 15, 2419-2428. doi: 10.1039/C5LC00180C

Morizane, R., Lam, A. Q., Freedman, B. S., Kishi, S., Valerius, M. T., and Bonventre, J. V. (2015). Nephron organoids derived from human pluripotent stem cells model kidney development and injury. Nat. Biotechnol. 33, 1193-1200. doi: 10.1038/nbt.3392

Müller-Deile, J., Schenk, H., Schroder, P., Schulze, K., Bolaños-Palmieri, P., Siegerist, F., et al. (2019). Circulating factors cause proteinuria in parabiotic zebrafish. Kidney Int. 96, 342-349. doi: 10.1016/j.kint.2019.02.013

Musah, S., Mammoto, A., Ferrante, T. C., Jeanty, S. S. F., Hirano-Kobayashi, M., Mammoto, T., et al. (2017). Mature induced-pluripotent-stem-cell-derived human podocytes reconstitute kidney glomerular-capillary-wall function on a chip. Nat. Biomed. Eng. 1:0069. doi: 10.1038/s41551-017-0069

Musso, C. G., and Oreopoulos, D. G. (2011). Aging and physiological changes of the kidneys including changes in glomerular filtration rate. Nephron Physiol. 119(Suppl. 1), 1-5. doi: 10.1159/000328010

Nishinakamura, R. (2019). Human kidney organoids: progress and remaining challenges. Nat. Rev. Nephrol. 15, 613-624. doi: 10.1038/s41581-019-0176-x

Öberg, C. M., and Rippe, B. (2013). Quantification of the electrostatic properties of the glomerular filtration barrier modeled as a charged fiber matrix separating anionic from neutral Ficoll. Am. J. Physiol. Renal Physiol. 304, F781-F787. doi: 10.1152/ajprenal.00621.2012

Perico, L., Conti, S., Benigni, A., and Remuzzi, G. (2016). Podocyte-actin dynamics in health and disease. Nat. Rev. Nephrol. 12, 692-710. doi: 10.1038/ nrneph.2016.127

Petrosyan, A., Cravedi, P., Villani, V., Angeletti, A., Manrique, J., Renieri, A., et al. (2019). A glomerulus-on-a-chip to recapitulate the human glomerular filtration barrier. Nat. Commun. 10:3656. doi: 10.1038/s41467-019-11577-Z

Pozzi, A., Jarad, G., Moeckel, G. W., Coffa, S., Zhang, X., Gewin, L., et al. (2008). Betal integrin expression by podocytes is required to maintain glomerular structural integrity. Dev. Biol. 316, 288-301. doi: 10.1016/j.ydbio.2008.01.022

Quinlan, C., and Rheault, M. N. (2021). Genetic basis of type IV collagen disorders of the kidney. Clin. J. Am. Soc. Nephrol. 13:CJN.19171220. doi: $10.2215 /$ cjn. 19171220

Ramnath, R. D., and Satchell, S. C. (2020). Glomerular endothelial cells: assessment of barrier properties in vitro. Methods Mol. Biol. 2067, 145-151. doi: 10.1007/978-1-4939-9841-8_11

Rayner, S. G., Howard, C. C., Mandrycky, C. J., Stamenkovic, S., Himmelfarb, J., Shih, A. Y., et al. (2021). Multiphoton-guided creation of complex organspecific microvasculature. Adv. Healthc. Mater. e2100031. doi: 10.1002/ adhm.202100031 [Epub ahead of print]. 
Rennke, H. G., Cotran, R. S., and Venkatachalam, M. A. (1975). Role of molecular charge in glomerular permeability. Tracer studies with cationized ferritins. J. Cell Biol. 67, 638-646. doi: 10.1083/jcb.67.3.638

Rennke, H. G., and Venkatachalam, M. A. (1979). Glomerular permeability of macromolecules. Effect of molecular configuration on the fractional clearance of uncharged dextran and neutral horseradish peroxidase in the rat. J. Clin. Invest. 63, 713-717. doi: 10.1172/JCI109354

Ron, A., Azeloglu, E. U., Calizo, R. C., Hu, M., Bhattacharya, S., Chen, Y., et al. (2017). Cell shape information is transduced through tension-independent mechanisms. Nat. Commun. 8:2145. doi: 10.1038/s41467-017-02218-4

Satchell, S. C., and Braet, F. (2009). Glomerular endothelial cell fenestrations: an integral component of the glomerular filtration barrier. Am. J. Physiol. Renal Physiol. 296, F947-F956. doi: 10.1152/ajprenal.90601.2008

Satchell, S. C., Tasman, C. H., Singh, A., Ni, L., Geelen, J., von Ruhland, C. J., et al. (2006). Conditionally immortalized human glomerular endothelial cells expressing fenestrations in response to VEGF. Kidney Int. 69, 1633-1640. doi: $10.1038 /$ sj.ki.5000277

Satchell, S. C., and Tooke, J. E. (2008). What is the mechanism of microalbuminuria in diabetes: a role for the glomerular endothelium? Diabetologia 51, 714-725. doi: 10.1007/s00125-008-0961-8

Schiffer, M., Teng, B., Gu, C., Shchedrina, V. A., Kasaikina, M., Pham, V. A., et al. (2015). Pharmacological targeting of actin-dependent dynamin oligomerization ameliorates chronic kidney disease in diverse animal models. Nat. Med. 21, 601-609. doi: 10.1038/nm.3843

Schlondorff, D., and Banas, B. (2009). The mesangial cell revisited: no cell is an island. J. Am. Soc. Nephrol. 20, 1179-1187. doi: 10.1681/ASN.2008050549

Sharmin, S., Taguchi, A., Kaku, Y., Yoshimura, Y., Ohmori, T., Sakuma, T., et al. (2016). Human induced pluripotent stem cell-derived podocytes mature into vascularized glomeruli upon experimental transplantation. J. Am. Soc. Nephrol. 27, 1778-1791. doi: 10.1681/ASN.2015010096

Singh, A., Friden, V., Dasgupta, I., Foster, R. R., Welsh, G. I., Tooke, J. E., et al. (2011). High glucose causes dysfunction of the human glomerular endothelial glycocalyx. Am. J. Physiol. Renal Physiol. 300, F40-F48. doi: 10.1152/ajprenal.00103.2010

Singh, A., Satchell, S. C., Neal, C. R., McKenzie, E. A., Tooke, J. E., and Mathieson, P. W. (2007). Glomerular endothelial glycocalyx constitutes a barrier to protein permeability. J. Am. Soc. Nephrol. 18, 2885-2893. doi: 10.1681/asn.2007010119

Sison, K., Eremina, V., Baelde, H., Min, W., Hirashima, M., Fantus, I. G., et al. (2010). Glomerular structure and function require paracrine, not autocrine, VEGF-VEGFR-2 signaling. J. Am. Soc. Nephrol. 21, 1691-1701. doi: 10.1681/ ASN.2010030295

Slater, S. C., Beachley, V., Hayes, T., Zhang, D., Welsh, G. I., Saleem, M. A., et al. (2011). An in vitro model of the glomerular capillary wall using electrospun collagen nanofibres in a bioartificial composite basement membrane. PLoS One 6:e20802. doi: 10.1371/journal.pone.0020802

Slater, S. C., Ramnath, R. D., Uttridge, K., Saleem, M. A., Cahill, P. A., Mathieson, P. W., et al. (2012). Chronic exposure to laminar shear stress induces Kruppel-like factor 2 in glomerular endothelial cells and modulates interactions with co-cultured podocytes. Int. J. Biochem. Cell Biol. 44, 1482-1490. doi: 10.1016/j.biocel.2012.05.020

Sobreiro-Almeida, R., Melica, M. E., Lasagni, L., Romagnani, P., and Neves, N. M. (2021). Retinoic acid benefits glomerular organotypic differentiation from adult renal progenitor cells in vitro. Stem Cell Rev. Rep. doi: 10.1007/ s12015-021-10128-8 [Epub ahead of print].

St John, P. L., and Abrahamson, D. R. (2001). Glomerular endothelial cells and podocytes jointly synthesize laminin-1 and -11 chains. Kidney Int. 60, 1037-1046. doi: 10.1046/j.1523-1755.2001.0600031037.x

Sun, Y. B., Qu, X., Zhang, X., Caruana, G., Bertram, J. F., and Li, J. (2013). Glomerular endothelial cell injury and damage precedes that of podocytes in adriamycin-induced nephropathy. PLoS One 8:e55027. doi: 10.1371/journal. pone. 0055027

Sutherland, R. (1988). Cell and environment interactions in tumor microregions: the multicell spheroid model. Science 240, 177-184. doi: 10.1126/ science. 2451290

Taguchi, A., Kaku, Y., Ohmori, T., Sharmin, S., Ogawa, M., Sasaki, H., et al. (2014). Redefining the in vivo origin of metanephric nephron progenitors enables generation of complex kidney structures from pluripotent stem cells. Cell Stem Cell 14, 53-67. doi: 10.1016/j.stem.2013.11.010
Takasato, M., Er, P. X., Becroft, M., Vanslambrouck, J. M., Stanley, E. G., Elefanty, A. G., et al. (2014). Directing human embryonic stem cell differentiation towards a renal lineage generates a self-organizing kidney. Nat. Cell Biol. 16, 118-126. doi: 10.1038/ncb2894

Takasato, M., Er, P. X., Chiu, H. S., Maier, B., Baillie, G. J., Ferguson, C., et al. (2015). Kidney organoids from human iPS cells contain multiple lineages and model human nephrogenesis. Nature 526, 564-568. doi: 10.1038/nature15695

Tanigawa, S., Islam, M., Sharmin, S., Naganuma, H., Yoshimura, Y., Haque, F., et al. (2018). Organoids from nephrotic disease-derived iPSCs identify impaired NEPHRIN localization and slit diaphragm formation in kidney podocytes. Stem Cell Rep. 11, 727-740. doi: 10.1016/j.stemcr.2018.08.003

Tasnim, F., and Zink, D. (2012). Cross talk between primary human renal tubular cells and endothelial cells in cocultures. Am. J. Physiol. Renal Physiol. 302, F1055-F1062. doi: 10.1152/ajprenal.00621.2011

Tryggvason, K., Zhou, J., Hostikka, S. L., and Shows, T. B. (1993). Molecular genetics of Alport syndrome. Kidney Int. 43, 38-44. doi: 10.1038/ki.1993.8

USRDS (2020). 2020 USRDS Annual Data Report: Epidemiology of Kidney Disease in the United States. United States Renal Data System. Bethesda, MD: National Institutes of Health, National Institute of Diabetes and Digestive and Kidney Diseases.

Vallon, V. (2003). Tubuloglomerular feedback and the control of glomerular filtration rate. News Physiol. Sci. 18, 169-174. doi: 10.1152/nips.01442.2003 van den Berg, C. W., Ritsma, L., Avramut, M. C., Wiersma, L. E., van den Berg, B. M., Leuning, D. G., et al. (2018). Renal subcapsular transplantation of PSC-derived kidney organoids induces neo-vasculogenesis and significant glomerular and tubular maturation in vivo. Stem Cell Rep. 10, 751-765. doi: 10.1016/j.stemcr.2018.01.041

Vaughan, M. R., and Quaggin, S. E. (2008). How do mesangial and endothelial cells form the glomerular tuft? J. Am. Soc. Nephrol. 19, 24-33. doi: 10.1681/ ASN.2007040471

Veissi, S., Smeets, B., van den Heuvel, L. P., Schreuder, M. F., and Jansen, J. (2020). Nephrotic syndrome in a dish: recent developments in modeling in vitro. Pediatr. Nephrol. 35, 1363-1372. doi: 10.1007/s00467-019-4203-8

Vivante, A., and Hildebrandt, F. (2016). Exploring the genetic basis of earlyonset chronic kidney disease. Nat. Rev. Nephrol. 12, 133-146. doi: 10.1038/ nrneph.2015.205

Wang, J., Zhong, J., Yang, H. C., and Fogo, A. B. (2018a). Cross talk from tubules to glomeruli. Toxicol. Pathol. 46, 944-948. doi: 10.1177/01926 23318796784

Wang, L., Tao, T., Su, W., Yu, H., Yu, Y., and Qin, J. (2017). A disease model of diabetic nephropathy in a glomerulus-on-a-chip microdevice. Lab Chip 17, 1749-1760. doi: 10.1039/C7LC00134G

Wang, Y. Y., Tang, L. Q., and Wei, W. (2018b). Berberine attenuates podocytes injury caused by exosomes derived from high glucose-induced mesangial cells through TGFß1-PI3K/AKT pathway. Eur. J. Pharmacol. 824, 185-192. doi: 10.1016/j.ejphar.2018.01.034

Wilmer, M. J., Ng, C. P., Lanz, H. L., Vulto, P., Suter-Dick, L., and Masereeuw, R. (2016). Kidney-on-a-chip technology for drug-induced nephrotoxicity screening. Trends Biotechnol. 34, 156-170. doi: 10.1016/j.tibtech.2015.11.001

Woods, I. G., Kelly, P. D., Chu, F., Ngo-Hazelett, P., Yan, Y. L., Huang, H., et al. (2000). A comparative map of the zebrafish genome. Genome Res. 10, 1903-1914. doi: 10.1101/gr.10.12.1903

Wu, H., Uchimura, K., Donnelly, E. L., Kirita, Y., Morris, S. A., and Humphreys, B. D. (2018). Comparative analysis and refinement of human PSC-derived kidney organoid differentiation with single-cell transcriptomics. Cell Stem Cell 23, 869.e8-881.e8. doi: 10.1016/j.stem.2018.10.010

Wu, X., Gao, Y., Xu, L., Dang, W., Yan, H., Zou, D., et al. (2017). Exosomes from high glucose-treated glomerular endothelial cells trigger the epithelialmesenchymal transition and dysfunction of podocytes. Sci. Rep. 7:9371. doi: 10.1038/s41598-017-09907-6

Wu, X. M., Gao, Y. B., Cui, F. Q., and Zhang, N. (2016). Exosomes from high glucose-treated glomerular endothelial cells activate mesangial cells to promote renal fibrosis. Biol. Open 5, 484-491. doi: 10.1242/bio.015990

Xia, Y., Nivet, E., Sancho-Martinez, I., Gallegos, T., Suzuki, K., Okamura, D., et al. (2013). Directed differentiation of human pluripotent cells to ureteric bud kidney progenitor-like cells. Nat. Cell Biol. 15, 1507-1515. doi: 10.1038/ncb2872

Xie, R., Korolj, A., Liu, C., Song, X., Lu, R. X. Z., Zhang, B., et al. (2020). H-FIBER: microfluidic topographical hollow fiber for studies of glomerular filtration barrier. ACS Cent. Sci. 6, 903-912. doi: 10.1021/acscentsci.9b01097 
Yang, S. H., Choi, J. W., Huh, D., Jo, H. A., Kim, S., Lim, C. S., et al. (2017). Roles of fluid shear stress and retinoic acid in the differentiation of primary cultured human podocytes. Exp. Cell Res. 354, 48-56. doi: 10.1016/j. yexcr.2017.03.026

Yaoita, E., Yoshida, Y., Nameta, M., Takimoto, H., and Fujinaka, H. (2018). Induction of interdigitating cell processes in podocyte culture. Kidney Int. 93, 519-524. doi: 10.1016/j.kint.2017.06.031

Yuen, D. A., Stead, B. E., Zhang, Y., White, K. E., Kabir, M. G., Thai, K., et al. (2012). eNOS deficiency predisposes podocytes to injury in diabetes. J. Am. Soc. Nephrol. 23, 1810-1823. doi: 10.1681/ASN.2011121170

Zennaro, C., Rastaldi, M. P., Bakeine, G. J., Delfino, R., Tonon, F., Farra, R., et al. (2016). A nanoporous surface is essential for glomerular podocyte differentiation in three-dimensional culture. Int. J. Nanomed. 11, 4957-4973. doi: $10.2147 /$ Ijn.S110201

Zhang, T., Lih, D., Nagao, R. J., Xue, J., Berthier, E., Himmelfarb, J., et al. (2020). Open microfluidic coculture reveals paracrine signaling from human kidney epithelial cells promotes kidney specificity of endothelial cells. Am. J. Physiol. Renal Physiol. 319, F41-F51. doi: 10.1152/ajprenal.00069.2020

Zhao, H. J., Wang, S., Cheng, H., Zhang, M. Z., Takahashi, T., Fogo, A. B., et al. (2006). Endothelial nitric oxide synthase deficiency produces accelerated nephropathy in diabetic mice. J. Am. Soc. Nephrol. 17, 2664-2669. doi: 10.1681/ASN.2006070798
Zhong, F., Chen, H., Wei, C., Zhang, W., Li, Z., Jain, M. K., et al. (2014). Reduced Kruppel-like factor 2 expression may aggravate the endothelial injury of diabetic nephropathy. Kidney Int. 87, 382-395. doi: 10.1038/ ki.2014.286

Zhou, M., Zhang, X., Wen, X., Wu, T., Wang, W., Yang, M., et al. (2016). Development of a functional glomerulus at the organ level on a chip to mimic hypertensive nephropathy. Sci. Rep. 6:31771. doi: 10.1038/srep31771

Zhou, W., and Hildebrandt, F. (2012). Inducible podocyte injury and proteinuria in transgenic zebrafish. J. Am. Soc. Nephrol. 23, 1039-1047. doi: 10.1681/ ASN.2011080776

Conflict of Interest: The authors declare that the research was conducted in the absence of any commercial or financial relationships that could be construed as a potential conflict of interest.

Copyright (c) 2021 Ebefors, Lassén, Anandakrishnan, Azeloglu and Daehn. This is an open-access article distributed under the terms of the Creative Commons Attribution License (CC BY). The use, distribution or reproduction in other forums is permitted, provided the original author(s) and the copyright owner(s) are credited and that the original publication in this journal is cited, in accordance with accepted academic practice. No use, distribution or reproduction is permitted which does not comply with these terms. 